\title{
Image Contrast Enhancement by Scaling Reconstructed Approximation Coefficients using SVD Combined Masking Technique
}

\author{
${ }^{1}$ Sandeepa K S, ${ }^{2}$ Basavaraj N Jagadale \\ Department of Electronics \\ Kuvempu University \\ Karnataka, India \\ ${ }^{3}$ J S Bhat \\ Department of Physics \\ Karnataka University \\ Karnataka, India
}

\author{
${ }^{4}$ Mukund N Naragund \\ Department of Electronics \\ Christ University \\ Karnataka, India
}

\author{
${ }^{4}$ Panchaxri \\ Department of Electronics \\ SSA Govt First Grade College \\ Ballari, Karnataka, India
}

\begin{abstract}
The proposed method addresses the general issues of image contrast enhancement. The input image is enhanced by incorporating discrete wavelet transform, singular value decomposition, standard intensity deviation based clipped sub image histogram equalization and masking technique. In this method, low pass filtered coefficients of wavelet and its scaled version undergoes masking approach. The scale value is obtained using singular value decomposition between reconstructed approximation coefficients and standard intensity deviation based clipped sub image histogram equalization image. The masking image is added to the original image to produce a maximum contrast-enhanced image. The supremacy of the proposed method tested over other methods. The qualitative and quantitative analysis is used to justify the performance of the proposed method.
\end{abstract}

Keywords-Standard intensity deviation clipped sub image histogram equalization; discrete wavelet transform; singular value decomposition; masking technique

\section{INTRODUCTION}

The image enhancement has many practical applications. There has been continuous research on developing new algorithms for different applications. In an image analysis, intensity-based enhancement techniques like contrast enhancement help to improve the clarity of the image [1]. The intensity enhancement in an image has been realized through techniques based on the histogram, transform domain filtering and masking approaches. Histogram equalization (HE) or generalized histogram equalization (GHE) [2], are simple approaches as these techniques stretch the gray values effectively but give over enhancement. Some of the methods employed to preserve the brightness are, Bi-histogram, Brightness preserving bi-histogram equalization (BBHE) [3] and dualistic sub image histogram equalization (DSIHE) [4], but these methods fail to suppress noise artifacts. Exposurebased sub image histogram equalization (ESIHE) [5] is best suited for low exposure image but less fit to contrast enhancement of medical images.
The discrete cosine transform (DCT) domain, provide spectral separation [6]. However, there are few drawbacks in transforming the image using block DCT. Therefore, discrete wavelet transform (DWT) has found application in contrast enhancement [7]. Wavelet coefficients have inherent qualities such as sparsity and decomposition [8]. The DCT-SVD and DWT-SVD based illumination enhancement presented by updating singular value matrix of singular value decomposition (SVD). In these methods, the low-low subband of the input image is considered by applying DWT [9], [10].

Recent algorithms for contrast enhancement are based on masking techniques [11]-[14]. In these methods, filtered version of original image have internal scaling processes, and the drawback of these approaches is the use of fixed scale value, irrespective of the input image. The scale values are selected randomly.

In this paper, we tried to address over enhancement issue using effective sub image histogram equalization process. We tried careful rescaling of the reconstructed approximation coefficients using SVD approach to achieve better results. Also, the minute details of the image and sensitive edges are preserved with the help of masking approach.

This work partially motivated by Un-sharp masking technique [11], an excellent result of SVD in contrast enhancement [15] and standard intensity deviation based clipped sub image histogram equalization (SIDCSIHE) [16]. We have used SVD to select intensity information of the SIDCSIHE and reconstructed approximation coefficients. In this paper, we tried to improve upon our previous work [17], the paper is structured as follows: Section 2 contains the proposed method and in Section 3 we discuss experimental results. The conclusions are given in Section 4.

\section{PROPOSED METHODOLOGY}

The medical image analysis depends upon quality; therefore contrast enhancement is often desirable for 
interpretation and diagnosis. This paper presents an improved contrast and enhancement in image quality by using DWT, SIDCSIHE, and SVD along with masking approach.

\section{A. Discrete Wavelet Transformation}

The low contrast image is subjected to DWT. The DWT decomposes the image into frequency sub-bands, namely, low low (LL), low high (LH), high low ((HL) and high high (HH) subbands as shown in Fig. 1. The LL subband is approximation coefficients and contains illumination information and image features. The decomposed approximation coefficients are obtained from (1) and its reconstruction is achieved by using inverse discrete wavelet transformation (IDWT) as given by (2).

$$
\begin{aligned}
& W_{\varphi}\left(j_{0}, m, n\right)=\frac{1}{\sqrt{M N}} \sum_{x=0}^{M-1} \sum_{y=0}^{N-1} f(x, y) \varphi_{j_{0}, m, n}(x, y) \\
& f_{(x, y)}^{A}=\frac{1}{\sqrt{M N}} \sum_{m} \sum_{n} W_{\varphi}\left(j_{0}, m, n\right) \varphi_{j_{0}, m, n}(x, y)
\end{aligned}
$$

where, $\boldsymbol{W}_{\boldsymbol{\varphi}}\left(\boldsymbol{j}_{\mathbf{0}}, \boldsymbol{m}, \boldsymbol{n}\right)$ is the approximation coefficient, $\boldsymbol{f}(\boldsymbol{x}, \boldsymbol{y})$ is the input time domain image of size $\mathrm{M} \times \mathrm{N}$ with discrete variable $(\mathrm{x}, \mathrm{y}) \cdot \boldsymbol{\varphi}_{\boldsymbol{j}_{0}, \boldsymbol{m}, \boldsymbol{n}}(\boldsymbol{x}, \boldsymbol{y})$, is the scale function and $\boldsymbol{f}_{(\boldsymbol{x}, \boldsymbol{y})}^{\boldsymbol{A}}$ is the reconstructed approximation coefficient [2]. The wavelet function $(\mathrm{db} 1)$ is used for wavelet decomposition of image.

Fig. 2 shows the LL, LH, HL and HH subbands of the brain_MRI image. Here, the reconstructed LL subband intensity information is used to find the best scale with respect to the input image. The other subbands contain edge information and details of the image as it contains high frequency. The illumination improvement can be achieved by scaling the coefficients of the LL subband.

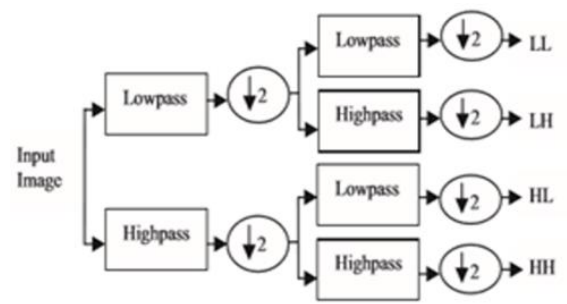

Fig. 1. Block diagram of DWT filter banks of level 1.

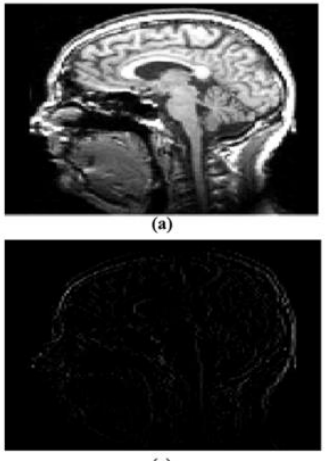

(c)
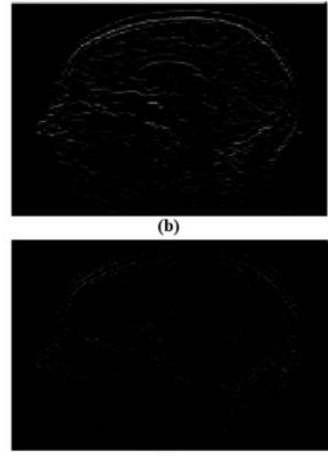

(d)

Fig. 2. Results of DWT decomposition of Brain_MRI image (a) Low Low subband; (b) Low High Sub band; (c) High Low subband; (d) High High subband.

\section{B. Standard Intensity Deviation based Clipped Sub Image Histogram Equalization (SIDCSIHE)}

Standard intensity deviation value is defined in (3) by using standard deviation function $\sigma$ as shown in (4) [18].

$$
\begin{aligned}
& X_{S I D V}=L *\left(1-\left(\frac{\sigma}{L}\right)\right) \\
& \sigma=\left(\frac{\sum_{i=1}^{L}\left(i-H_{\mu}\right)^{2} \times h(i)}{\sum_{i=1}^{L} h(i)}\right)^{1 / 2}
\end{aligned}
$$

The $H_{\mu}$, is a mean of the input image histogram is given by (5).

$$
H_{\mu}=\frac{\sum_{i=1}^{L} h(i) i}{\sum_{i=1}^{L} h(i)}
$$

where $h(i)$, is image histogram with its corresponding intensity $i$ and $L$ is its total number of gray levels.

To prevent over enhancement clipping histogram $T_{c}$ is calculated as in (6) and (7).

$$
\begin{array}{r}
T_{c}=\frac{1}{L} \sum_{K=1}^{L} h(k) \\
h_{c}(k)=T_{c} \text { for } h(k) \geq T_{c}
\end{array}
$$

$h_{c(k)}$, is the clipped histogram and it is computationally efficient [19]. Based on standard intensity deviation value $X_{S I D V}$, the clipped histogram is divided into two subimages $I_{\text {low }}$ and $I_{\text {up }}$ with ranges varying from 0 to $X_{S I D V}$ and $X_{S I D V}+1$ to $L-1$ respectively. The cumulative distribution function $C_{\text {low }}(i), C_{u p}(i)$ of each sub-image can be defined as

$$
\begin{aligned}
& C_{\text {low }}(i)=\sum_{i=0}^{X_{S I D} V} h_{c}(i) /_{N_{\text {low }}} \text { for } 0 \leq i \leq X_{S I D V} \\
& C_{u p}(i)=\sum_{i=X_{S I D V}+1}^{L-1} h_{c}(i) /_{N_{u p}} \text { for } X_{S I D V} \leq i \leq L-1
\end{aligned}
$$

where $N_{\text {low }}$ and $N_{\text {up }}$ are the total numbers of pixels in each sub-image. The histogram equalization is done individually for two sub-images using the transfer function $F(i)$ as expressed in (10) and sub-images are combined into one final image by the transfer function $F(i)$ [16].

$F(i)=\left\{\begin{array}{cl}X_{S I D V} * \quad C_{\text {low }} & \text { for } 0 \leq i \leq X_{S I D V} \\ \left(X_{S I D V}+1\right)+\left(L-X_{S I D V}+1\right) * C_{u p} & \text { for } X_{S I D V+1} \leq i \leq L-1\end{array}\right.$

The input image is preprocessed by SIDCSIHE that provides the equalized image and its intensity information helps to enhance the image contrast.

\section{Masking Technique}

In the masking approach, the reconstruction approximation coefficients and SIDCSIHE processed images are considered for masking formulation. The contrast of the reconstructed 
low pass signal is enhanced by using SVD and it helps to avoid information loss and better visual result. The SVD decompositions of the reconstruction approximation $\left(I_{1}\right)$ and SIDCSIHE images $\left(I_{2}\right)$ i.e. :

$$
\begin{aligned}
& I_{1}=U 1 \times S 1 \times V 1 \\
& I_{2}=U 2 \times S 2 \times V 2
\end{aligned}
$$

Here U1, V1, U2, and V2 are the orthogonal matrices identifies as left, right singular matrices of $I_{1}$ and $I_{2} . \mathrm{S} 1$ and $\mathrm{S} 2$ are sorted singular value of diagonal matrices, contain intensity information about the images $I_{1}$ and $I_{2}$. Any changes in the singular matrix will affect the image intensity. The most promising approach of the image contrast enhancement is altering the image intensity information stored in the singular value [20]. To modify the intensity information of the reconstructed approximation coefficients, the S1 matrix is altered by using proper weighting $(w)$ value. The highest singular value of $I_{1}$ and $I_{2}$ is used to calculate weighting value as shown in (13).

$$
w=\frac{\max (S 1)+\max (S 2)}{2 X \max (S 2)}
$$

The new reconstruction approximation $R A$ is obtained by modifying the S1 matrix as given in (14).

$$
R A=U 1 \times(w \times S 1) \times V 2
$$

The formulated mask, which gives the residual intensity information between $R A$ and reconstructed low pass signal. This mask image added to original image and output will be the contrast-enhanced image. The entire process of the method is shown in Fig. 3. The following steps the computational process of the proposed algorithm.

Step 1. Low contrast image was taken for processing.

Step 2. Equalize the image using standard intensity deviation based clipped sub image histogram equalization.

Step 3. Compute 1 level DWT to decompose the image into four sub-band.

Step 4. Perform reconstruction of the approximation coefficients.

Step 5. The SVD is applied to SIDCSIHE image and reconstructed approximation for getting $U 1 \times S 1 \times V 1$ and $U 2 \times S 2 \times V 2$ then $\max (S 1)$ and $\max (S 2)$ obtained.

Step 6. Calculate weighting value w by using (13).

Step 7. New reconstruction approximation coefficient is obtained by $R A=U 1 \times(w \times S 1) \times V 2$.

Step 8. Subtract the RA from reconstruction approximate matrix [mask].

Step 9. Add the mask with original image to get enhanced output image.

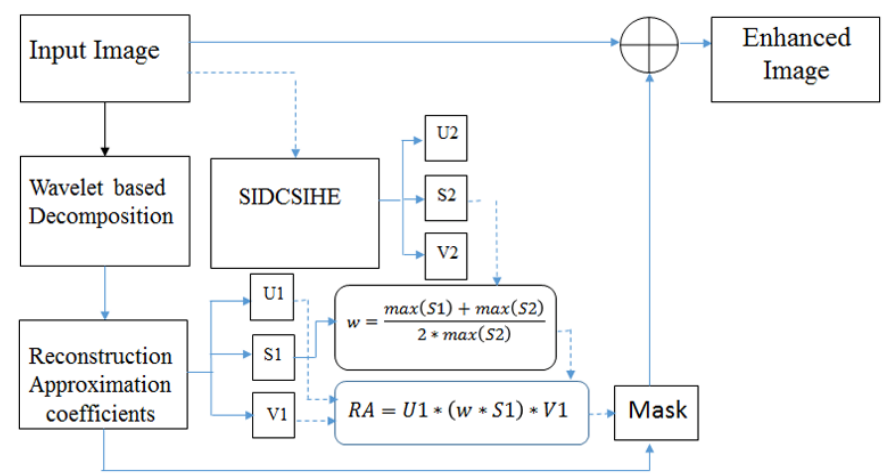

Fig. 3. Block diagram of the proposed method.

\section{EXPERIMENTAL RESULTS}

The supremacy of the proposed method is illustrated by comparing both qualitative and quantitative analysis with well-known existing methods like HE, BBHE, DSIHE, ESIHE. As far as qualitative analysis is concerned, the performance of the proposed method is judged by visual quality inspection. As far as quantitative analysis of the proposed method is measured in terms of AMBE, entropy, PSNR and SSIM values and are compared with other methods.

The proposed algorithm performance is evaluated using (15)-(18). Given that $\mathrm{MN}$ is the size of the image, $I_{\text {in }}$ is input image, $I_{e}$ the enhanced image, $\operatorname{Max}_{i n}$ the maximum possible pixel value of the input image. The PSNR is representing a measure of the peak error between the input and enhanced image.

$$
\begin{aligned}
& M S E=\frac{1}{(M N)} \sum_{i=1}^{M} \sum_{j=1}^{N}\left(I_{i n}(i, j)-I_{e}(i, j)\right)^{2} \\
& P S N R=10 \log _{10}\left(\frac{M a x_{i n}{ }^{2}}{M S E}\right) \\
& A M B E=\left|I_{i n}-I_{e}\right|
\end{aligned}
$$

Entropy means average information content and is defined in (18).

$$
\operatorname{Entropy}(p)=-\sum_{k=0}^{L-1} p(k) \log p(k)
$$

Where $p(k)$, is probability density function at the intensity level $k$ and $L$ is the total number of gray levels of the image.

\section{A. Qualitative Analysis}

The performance of the proposed method is well analyzed with medical images (Brain_MRI, Face X-ray, MRI, Ribs XRay) ${ }^{1}$ and mammogram images (mdb011, mdb013, mdb209, mdb211, mdb213) ${ }^{2}$.

Fig. 4(a-f), (g-l), (m-r), (s-x) are the results of the proposed method steps from the input image to the contrast-enhanced

\footnotetext{
${ }^{1}$ https://medpix.nlm.nih.gov/home

${ }^{2}$ http://www.mammoimage.org/databases
} 
image of (brain_MRI, Ribs X-Ray, mdb013, mdb212). The enhancement provided by SIDCSIHE method as shown in Fig. 4(b, h, n, t). Fig. 4(c, i, o, u) are reconstructed approximation coefficients, it contains necessary illumination information. The scaled reconstructed LL subband based SVD method can be seen in Fig. 4(d, j, p, v). The mask image contains residual intensity information clearly observed in Fig. 4(e, k, q, w). In Fig. 4(q), (w) shows the edge information and extracted intensity residual information. Fig. 4(f, l, r, x) are resultant, contrast-enhanced image with better visual quality than the original image.

The pleasant effect in the appearance of the contrastenhanced image can be seen in the face X-ray image as in Fig. 5. The HE, BBHE, DSIHE are over enhanced. The proposed image has good contrast enhancement result over ESIHE image.

The MRI image in Fig. 6 is the proposed method that yields contrast-enhanced image. The HE, DSIHE shows overenhancement and BBHE is dark in nature.

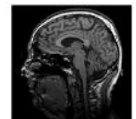

(a)

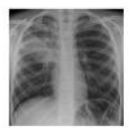

(g)

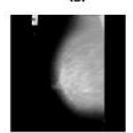

(m)

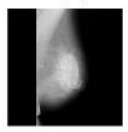

(s)

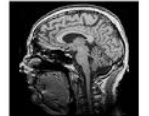

(b)

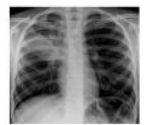

(h)

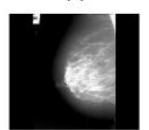

(n)

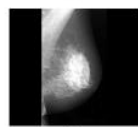

(t)

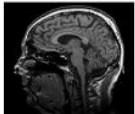

(c)

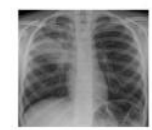

(i)

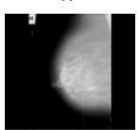

(o)

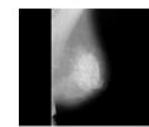

(u)

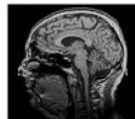

(d)

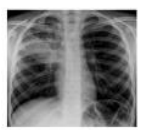

(j)

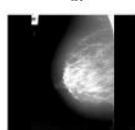

(p)

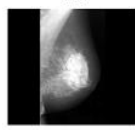

(v)

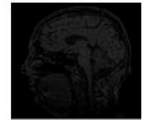

(e)

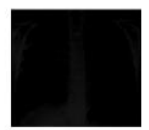

(k)

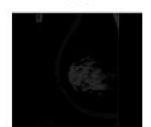

(q)

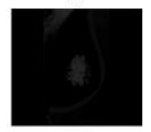

(w)

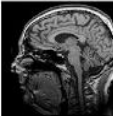

(f)

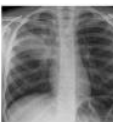

(I)

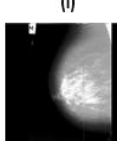

(r)

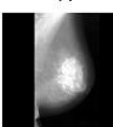

(x)
Fig. 4. Results of different stages of proposed method (a,g,m,s) input images; (b,h,n,t) SIDCSIHE ; (c, i, o,u) Reconstructed Approximation coefficient; (d,j,p,s) RA image (e, k,q,w) Mask image; (f,l,r,x) Output image.

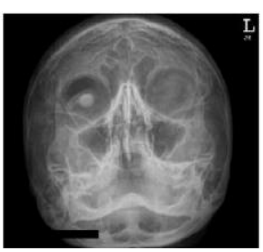

(a)

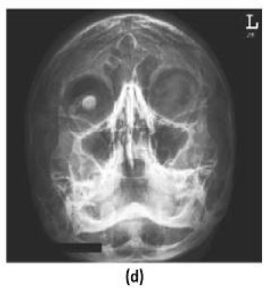

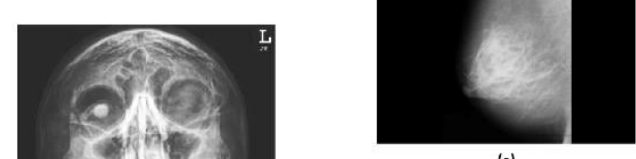

(d)

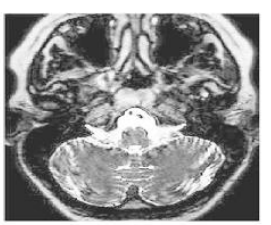

(b)

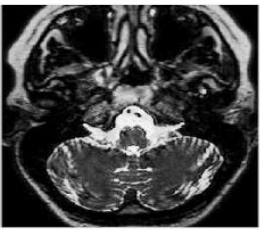

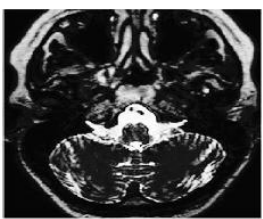

(c)

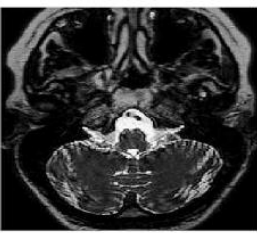

(f)
Fig. 6. Results of different methods for the MRI image (a) Low contrast input image; (b) HE; (c) BBHE (d) DSIHE; (e) ESIHE; (f) proposed method.

The analysis of the mammogram image (Fig. 7 and 8) supremacy of the proposed method is to show normal fatty tissue, dense breast tissue and infected area clearly.

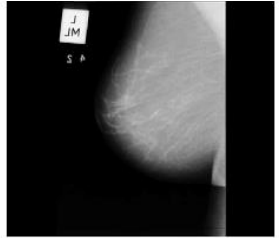

(a)

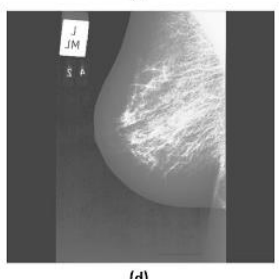

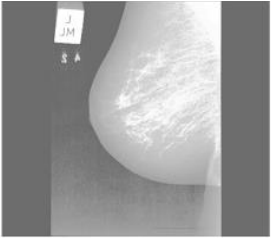

(b)

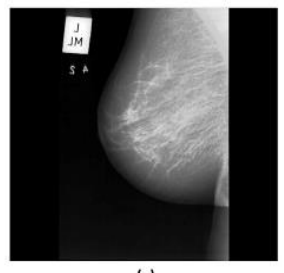

(e)

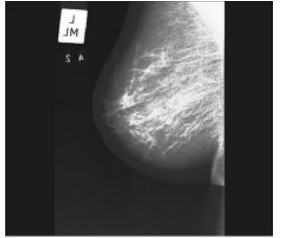

(c)

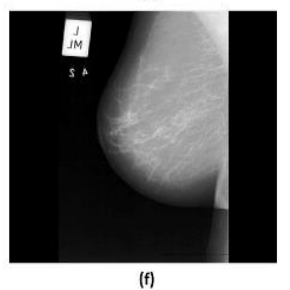

Fig. 7. Results of different methods for the mammogram (mdb011) (a) Low contrast input image; (b) HE; (c) BBHE (d) DSIHE; (e) ESIHE; (f) proposed (a)
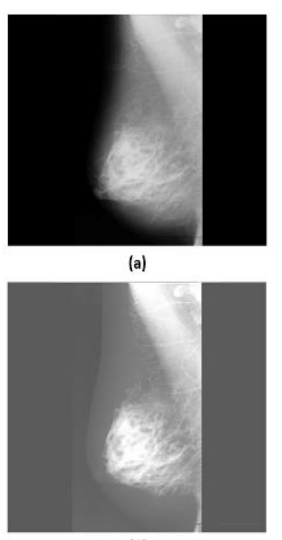
method.

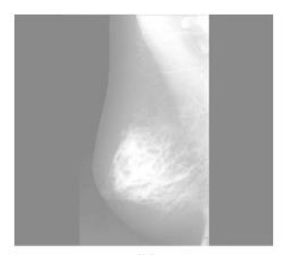

(b)

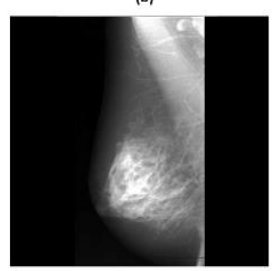

(e)

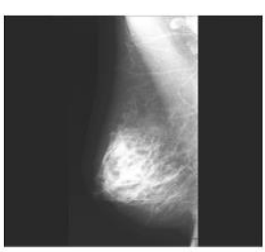

(c)

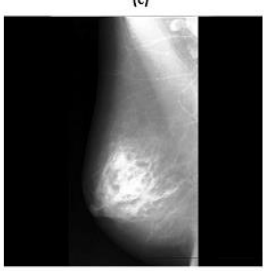

(f)
Fig. 8. Results of different methods for the mammogram (mdb211) (a) Low contrast input image; (b) HE; (c) BBHE (d) DSIHE; (e) ESIHE; (f) proposed method.

As compared to other methods in Fig. 7, proposed method clearly highlights the required region. The $\mathrm{HE}$ and DSIHE images are over enhanced. The dark image obtained by BBHE method. In Fig. 8, HE, BBHE and DSIHE are over enhanced 
and proposed method image provided acceptable visual information than the input image.

\section{B. Quantitative Analysis}

To evaluate the performance of the proposed method, quantitative analysis has studied in terms of AMBE, entropy, PSNR, and SSIM.

The quality of the proposed method is provided by AMBE values, which is tabulated in Table I. The average AMBE value lesser than other contrast enhancement technique shows the quality of the contrast improvement. The entropy values of all the input image are tabulated in Table II. The highest entropy value indicates more information content of the image. As compared to HE, BBHE, DSIHE, ESIHE, the proposed has greater entropy value.

The SSIM values also encourage the performance of the proposed method is shown in Fig. 9. To check noise artifacts produced and over enhancement, PSNR values of the enhancement results are compared and shown in Fig. 10. The average PSNR value produced by the proposed method for all images is greater than HE, BBHE, DSIHE, and ESIHE.

TABLE I. AMBE VALUES OF THE COMPARED METHODS FOR CONTRAST ENHANCEMENT

\begin{tabular}{|l|l|l|l|l|l|}
\hline $\begin{array}{l}\text { Test } \\
\text { Images }\end{array}$ & HE & BBHE & DSIHE & ESIHE & PROPOSED \\
\hline Brain_MRI & 89.529 & 29.611 & 31.566 & 24.019 & $\mathbf{1 3 . 6 0 0}$ \\
\hline Face X-Ray & 57.059 & 36.221 & 22.554 & 5.242 & $\mathbf{1 . 9 8 7}$ \\
\hline MRI & 103.587 & 27.551 & 42.799 & 32.576 & $\mathbf{1 8 . 7 5 4}$ \\
\hline Ribs X-Ray & 11.601 & 9.941 & 4.390 & 2.860 & 4.498 \\
\hline mdb011 & 103.552 & 21.866 & 62.995 & 4.279 & 4.307 \\
\hline mdb013 & 118.487 & 27.789 & 74.376 & 6.045 & $\mathbf{4 . 3 1 6}$ \\
\hline mdb209 & 102.731 & 25.904 & 50.772 & 3.883 & 4.375 \\
\hline mdb211 & 112.495 & 27.558 & 68.075 & 4.377 & 4.402 \\
\hline mdb212 & 114.276 & 24.981 & 69.593 & 4.136 & 4.309 \\
\hline Average & 90.369 & 25.713 & 47.458 & 9.713 & $\mathbf{6 . 7 2 8}$ \\
\hline
\end{tabular}

TABLE II. ENTROPY VALUES OF THE COMPARED METHODS FOR CONTRAST ENHANCEMENT

\begin{tabular}{|l|l|l|l|l|l|}
\hline Test Images & HE & BBHE & DSIHE & ESIHE & PROPOSED \\
\hline Brain_MRI1 & 5.021 & 5.859 & 5.886 & 6.161 & $\mathbf{6 . 6 5 6}$ \\
\hline Face X-Ray & 4.991 & 6.113 & 6.095 & 6.298 & $\mathbf{6 . 5 2 2}$ \\
\hline MRI & 5.045 & 5.551 & 5.613 & 5.817 & $\mathbf{6 . 2 0 5}$ \\
\hline Ribs X-Ray & 5.980 & 7.157 & 7.173 & 7.216 & $\mathbf{7 . 4 4 9}$ \\
\hline mdb011 & 3.742 & 4.371 & 4.318 & 4.587 & $\mathbf{4 . 7 3 6}$ \\
\hline mdb013 & 3.001 & 3.780 & 3.722 & 3.970 & $\mathbf{4 . 1 2 5}$ \\
\hline mdb209 & 3.842 & 4.758 & 4.667 & 4.943 & $\mathbf{5 . 1 1 0}$ \\
\hline mdb211 & 3.107 & 4.146 & 4.098 & 4.329 & $\mathbf{4 . 6 4 8}$ \\
\hline mdb212 & 3.223 & 4.038 & 4.022 & 4.240 & $\mathbf{4 . 4 5 0}$ \\
\hline Average & 4.217 & 5.086 & 5.066 & 5.285 & $\mathbf{5 . 5 4 5}$ \\
\hline
\end{tabular}

www.ijacsa.thesai.org

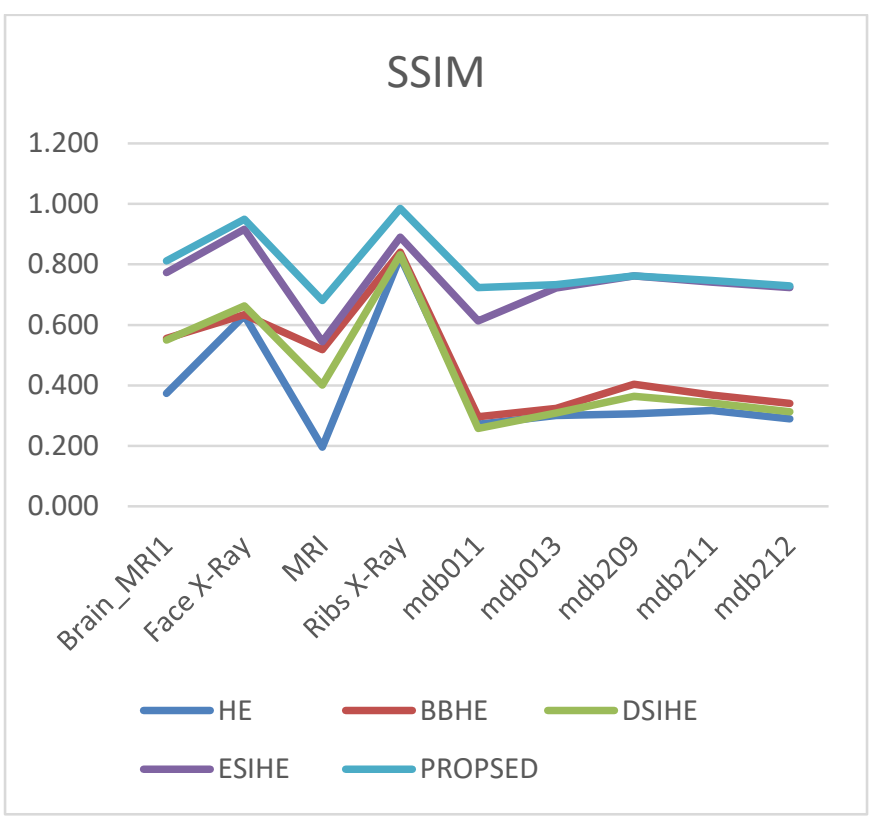

Fig. 9. SSIM values of the compared methods for contrast enhancement.

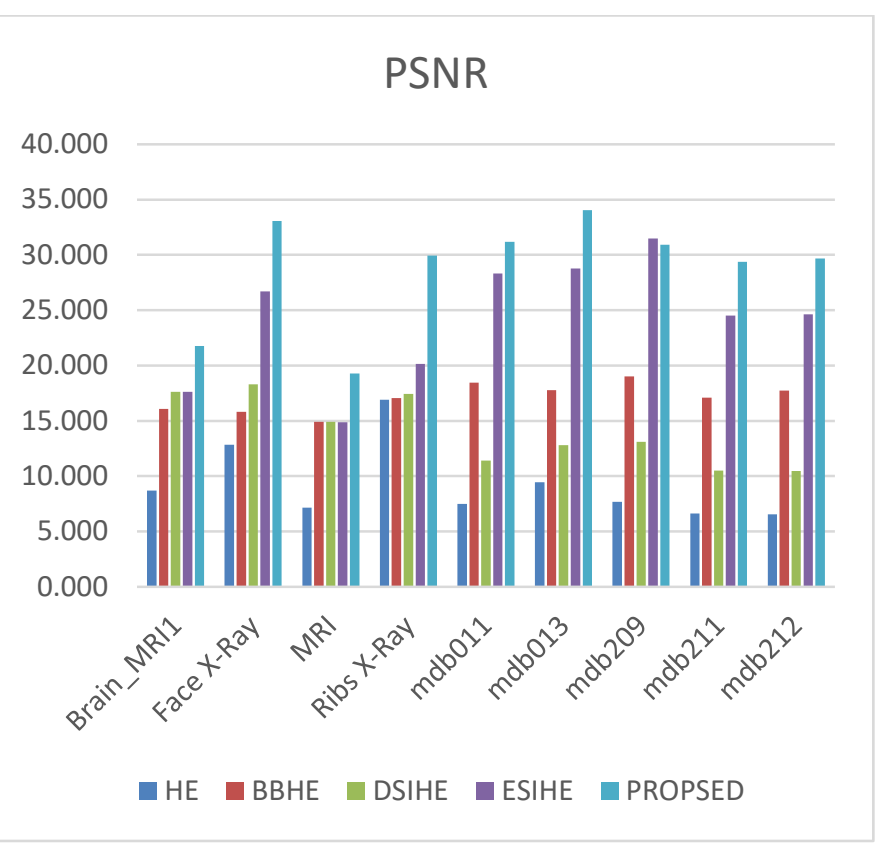

Fig. 10. PSNR values of the compared methods for contrast enhancement.

\section{Summary of Analysis and Discussion}

The objective of this paper is to obtain improved contrast enhancement by incorporating SIDCSIHE, DWT, SVD and masking technique. The main advantage of this paper is applying SVD technique effectively on SIDCSIHE image and reconstruction of LL image. The masking helps in providing residual information, which can be added to the original image for achieving maximum contrast enhancement. The proposed algorithm has been implemented in MatlabR2010, running on Windows PC with Intel i3 at 1.90GHZ and 4GB RAM. 
After qualitative and quantitative analysis it can be concluded that

- Proposed method improves image contrast in comparison to other methods.

- The proposed method provides minimum AMBE vale, it shows the quality of contrast improvement.

- The proposed method provides the highest entropy among other methods, it shows the richness of details.

- Proposed method provide better PSNR and SSIM value, it shows over enhancement control and structural similarity.

- Proposed method produces an image with good contrast enhancement.

\section{CONCLUSION}

In this paper, we tried to develop a method to address general issues of the image contrast enhancement. The proposed method effectively used masking approach to collect the sensitive edge information as residual intensity information. The method uses standard intensity deviation based clipped sub image histogram equalization to enhance contrast by controlling over enhancement. The SVD method helps to modify the intensity information. The experimental result shows the robustness of the proposed method as compared to the existing algorithm for the variety of images.

However, the proposed method does not consider the problem of noise enhancement, as the DWT method is shift variant. The work can be improved by using shift invariance transform along with the noise eliminating filter, to suppress the noise during enhancement. We have considered SVD to calculate scale value. The optimized algorithm can incorporate to find scale value. In this work contrast enhancement is achieved, this can be extended into resolution enhancement by using better interpolation algorithm.

\section{ACKNOWLEDGMENT}

This research work is supported by UGC-MRP, New Delhi, India.

\section{REFERENCES}

[1] Araujo, A.F.D. New artificial life model for image enhancement. Expertsyst 2014, 41, 5892-5906.

[2] Gonzalez, R.C.; Woods, R. Digital Image Processing, 3rd ed.; Pearson, INDIA, 2014, pp. 144-166.

[3] Y. T.Kim, "Contrast Enhancement using Brightness preserving bihistogram equalization", IEEE Trans. Consumer Electron, (1997), 43: $1-8$.
[4] Y.Wan, Q. Chen and B. M. Zhang. "Image enhancement based on equal area dualistic sub image histogram equalization method", IEEE Trans. Consumer Electron, 1999, 68-75.

[5] Kuldeep sigh, Rajiv Kapoor, "Image enhancement using Exposure-based Sub Image Histogram Equalization", pattern recognition letters 26 (2014) 10-14.

[6] Weeks A R, Sartor LJ, Myler H R. Histogram specification of 24-bit color images in the color difference $(\mathrm{C}-\mathrm{Y})$ color space. Proc.SPIE1999; 3646:319-29.

[7] Mukherjee J, Mitra S K.Enhancement of color images by scaling the DCT coefficients. IEEE Trans ImageProcess 2008; 17(10):1783-94.

[8] Mallat G.Theory for multi-resolution signal decomposition: the wavelet representation.IEEE Trans Pattern AnalMach Intell1989;2(7):674-94.

[9] Bhandari A K, Gadde M, Kumar A, Singh, G K.Comparative analysis of different wavelet filters for low contrast and brightness enhancement of multispectral remote sensing images. In: Proceedings of the IEEE international conference on machine vision and image processing(MVIP),p.81-6; 2012.

[10] Bhandari A K, Kumar A, Padhy P K.Enhancement of lowcontrast satellite images using discrete cosine transform and singular value decomposition. WorldAcad Sci EngTechnol2011; 79:35-41.

[11] Guang Deng. A Generalized Unsharp Masking Algorithm. IEEE TRANSACTIONS ON IMAGE PROCESSINGMay 2011, 20, 123-126.

[12] Ching Chung Yang. A modification for the mask-filtering approach by superposing anisotropic derivatives in an image. Optik - International Journal for Light and Electron OpticsSep 2011, 122, 1684-1687, issue 18.

[13] Polesel, A.; Ramponi,g.; Mathews, V.J. Image enhancement via adaptive unsharp masking. ", IEEE Transactions on Image Processing Mar 2000, 9, issue 3.

[14] V.S. Hari, V.P.J.Raj, R.Gopikakumari, Unsharp masking using quadratic filter for the enhancement of fingerprints in noisy background, PatternRecognit.46 (2013)3198-3207.

[15] Bhandaria A k, Sonia V. H, Kumaran A, Singhb G K.Cuckoo search algorithm based satellite image contrast and brightness enhancement using DWT-SVD. ISA Transactions 2014, 53, 1286-1296.

[16] Sandeepa K S, Basavaraj N Jagadale and J S Bhat, "Standard Intensity Deviation Approach based Clipped Sub Image Histogram Equalization Algorithm for Image Enhancement" International Journal of Advanced Computer Science and Applications(IJACSA), 9(1), 2018. http://dx.doi.org/10.14569/IJACSA.2018.090116

[17] Sandeepa K S, Basavaraj N Jagadale, J S Bhat, Naveen Kumar R, Mukund $\mathrm{n}$ naragund, panchaxri, "Image contrast enhancement using DWT-SVD based masking technique", IEEE explore, (ICCES 2017), ISBN:978-1-5090-5013-0.

[18] Shin-Chia Huang and Chien-Hui Yeh. "Image contrast enhancement for preserving mean brightness without losing image features".Engineering Application of Artificial Intelligence,( 2013), 26:1487-1492.

[19] C.H. Ooi and N.S.P. Kong, "Ibrahmin, H. Bi-histogram equalization with plateau limit for digital image enhancement", IEEE Trans. Consumer Electron ,( 2009),55 (4), 2072-2080.

[20] H. Demirel, C. Ozcinar, G. Anbarjafari, Satellite image contrast enhancement using discrete wavelet transform and singular value decomposition, IEEE Geosci. Remote Sens. Lett. 7 (April (2)) (2010). 